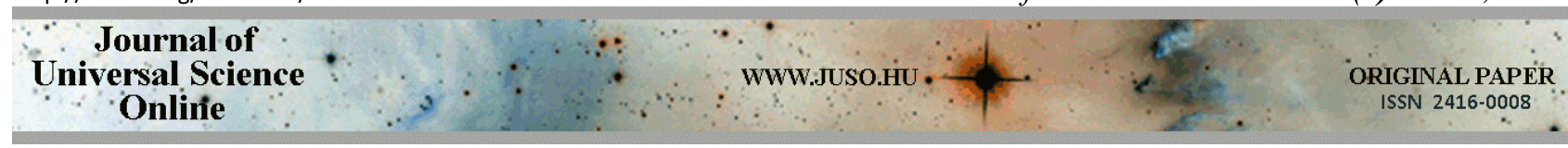

\title{
General symmetry properties of incommensurately modulated crystals described by projective representations of line groups
}

\author{
Ágnes BÁLINT ${ }^{1, *}$, István NIKOLÉNYI ${ }^{2}$ and Csaba MÉSZÁROS ${ }^{2}$ \\ ${ }^{1}$ Institute of Environmental Engineering, Óbuda University, Doberdó u.6., Budapest, H-1034, Hungary \\ ${ }^{2}$ Department of Physics and Process Control, Szent István University, Páter K.u.1., Gödöllő, H-2103, Hungary \\ ${ }^{*}$ Corresponding Author; e-mail: balint.agnes@rkk.uni-obuda.hu
}

Received: 7 November 2015 / Revised: 1 December 2015 / Accepted: 29 December 2015

Keywords: Line groups, Incommensurate structures, Projective representations

\begin{abstract}
After a detailed investigation of some fundamental general orthogonal symmetry properties of incommensurate condensed systems, the line groups are applied for describing the structure of such modulated crystals. It is demonstrated, that use of projective representations of these groups may give a more refined description of such structures and significantly extends applicability of the diffuse scattering formalism.
\end{abstract}

\section{Introduction}

It is well-known, that applications of the exact symmetry theory of discrete crystallinetype quasi-one-dimensional $(Q 1 D)$ systems [1] has been playing, and plays even nowadays a role of crucial importance in both experimental and theoretical investigations of different types of condensed matter systems. Throughout this paper we will call the relevant symmetry groups line groups, as it has been widely accepted in literature e.g. [2], [3]. From the most important applications of them, structural investigation of chain molecules by X-ray diffraction has been known for decades e.g. [4], and played a crucial role in experimental studies at revealing of fundamental structural properties of the DNA-molecule [5-6]. An exact and detailed reformulation of the precise abstract algebraic theory of line groups [2], [7] and their irreducible representations [8-9] came later. A detailed and up-to-date description of this symmetry technique can be found in a recent monograph by Damnjanović and Milošević [3].

A relatively recent important research area, where the application of the line group theory has been demonstrated to be very useful is the investigation of carbonnanotubes, where after a study of fundamental importance by Damnjanović and co-workers [10] numerous useful applications of the exact representation theory of the symmetry groups of $Q 1 D$ systems has been succesfully demonstrated [11]. Among them, calculation of diffraction intensities on the base of the symmetries of carbon nanotubes e.g. [12] also represents a particularly important and promising technique from the point of view of future applications.

Besides, some very general types of structural phase transitions (including those resulting in incommensurately modulated crystal structures) have also been investigated in detail - but within frame of the mean field 


Journal of
Universal Science
Online

approximation (MFA) only - by the theory of line groups and their irreducible representations [13-18].

Finally, we would like also to mention here the crucial domain of biopolymers, where beyond the newest modelling results of the elementary excitations (which are in general case of strongly nonlinear character) of the DNA molecules in both quantummechanical [19] and classical mechanical sense, (i.e. using tools of the classical elasticity theory [20]), the alpha-helix protein molecule is also one of the best-known examples, whose soliton-type elementary excitations represent active research topic for decades e.g. [21-22], but which have been treated without any detailed application of the theory of line groups and their irreducible representations.
Therefore, it may be stated, that since their discovery, the mathematical formalism of line groups plays a role of continuously increasing importance in various branches of the condensed matter theory, despite of the fact, that there are many intensively studied, but still open research domains where they have not been applied in detail, e.g. in the theory of Peierls' transitions, basic symmetries of liquid crystals and contemporary theories of biopolymers, just to mention a few. In the present work, the author intend to demonstrate, that projective representations of line groups represent a very suitable tool for further refinements of the existing mathematical formalism of scattering processes at $Q 1 D$ systems and propose on this base a novel, more refined description of the diffuse X-ray scattering formalism.

\section{A BRIEF DISCUSSION OF ESSENTIAL FEATURES OF THE EXACT ABSTRACT SYMMETRY AND REPRESENTATION THEORY OF Q1D SYSTEMSAND THEIR APPLICATIONS IN THE EVALUATION OF SCATTERING PROCESSES}

\section{Basic elements of the theory of line groups and their irreducible representations}

\section{The basic algebraic structure}

According to the definition, the complete set of symmetry transformations leaving invariant a $Q 1 D$ system belongs to one of the (discrete) infinitely many line groups gathered into 13 families [2]. (We mention here in advance, that the irreducible representation $D^{(\mu)}(L)$ of a full line group $L$ can be obtained

\section{Application of projective representations}

Although some applications of the projective representations of crystallographic point groups in solid state physics are known for decades [25-26] (their relevance in the case of classical description within frame of the Schönfliess-Fedorov Shubnikov theory of crystallographic space groups was also pointed out by Landau and Lifshitz [27]), they are completely absent even from the most from the irreducible representation of symmetry groups (i.e. point groups) of the motifs $D^{(v)}(P)$ by use of the induction technique elaborated in the theory of group representations and widely applied in solid state physics e.g. [23-24] and usually denoted by $\left.D^{(\mu)}(L)=D^{(v)}(P) \uparrow L\right)$.

complete works about applications of line groups in various types of structural investigations of condensed matter systems [3-4].

In the next section we will demonstrate following our own earlier basic result [17] (which may be considered as a simple first attempt to introduce the projective representations of line groups into exact 


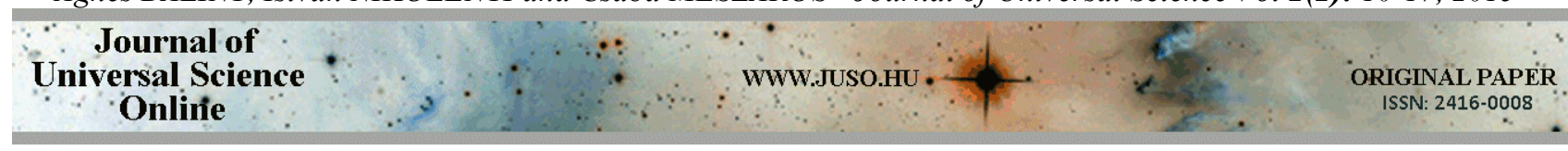

symmetry theory of incommensurately modulated crystals) - formalism of the projective representations of groups in order to refine the already existing mathematical formalism of the theory of invariants relevant for phase transitions resulting in different types of modulated crystal structures. The same symmetry method will also be applied in formalism of the applied Fourier-analysis necessary for describing the diffuse scattering processes from modulated structures of general type. Finally, we also think, that it must also be pointed out here, that the mathematical terminology emanating from line group theory (which may be considered as the most general algebraic formalism of fractional translations), is not to be confused directly with terminology connected to the most general theory of transport processes, known nowadays under name of anomalous diffusion theory, too (e.g. [28]), despite of the fact, that the latter one has also been topic of very serious and detailed symmetry analyses, too [29].

\section{A novel concept for applying the line group technique in the case of incommensurate systems}

In this section we will represent the essential result of the present study on the base of the general symmetry methods explained in [2] and [26]. Accordingly, if a line group $L$ is composed from line groups $L_{1}$ and $L_{2}$, which have the same translational subgroup $T$, then its relevant point group $P$ is in relation $P=P_{1} \wedge P_{2}$ with the point groups of its constituent line groups. Then, the following condition (explained by point group elements , $R$ " and fractional translations , $v$ ") must be satisfied:

$$
v_{2}+R_{2} v_{1}-R^{\prime} v_{2}=v^{\prime}+t, \quad t=0, \pm 1, \pm 2, \ldots
$$

where $\left(R_{1} \mid v_{1}\right)$ and $\left(R_{2} \mid v_{2}\right)$ are any coset representatives in $L_{1}$ and $L_{2}$, respectively (then, the relation $R^{\prime}=R_{2} R_{1} R_{2}^{-1}$ is also valid). Then, if we compare this condition with the one emanating from the general treatment of basic properties of the unimodular group in two dimensions (i.e. characterized by three (continuously varying!) parameters), defined by use of the elementary linear transformation formulae $g_{a} \equiv x^{\prime}=a_{1} x+a_{2}, g_{b} \equiv x^{\prime \prime}=b_{1} x^{\prime}+b_{2}$, it is represented by

$$
\xi \mapsto \frac{a_{1} \xi+a_{2}}{b_{1} \xi+b_{2}}
$$

(see also the Appendix in this respect). Then, the relevant most general conjugate transformation $g_{a} g_{b} g_{a}^{-1}$ from the unimodular group is realized by

$$
a_{1} x+a_{2} b_{1}-a_{1} b_{2}=x^{\prime}-b_{2} \text {. }
$$

Although the simultaneous application of the formulae (1-3) may explain the essential symmetry features of two different translationally invariant (with translational invariance in the same direction, but with generating elements, which can not be interrelated with integer numbers!) Q1D systems, this possible connection has not been applied in detail in the case of incommensurately modulated condensed matter systems. Therefore, the essential main new result of the present study is to fill this 


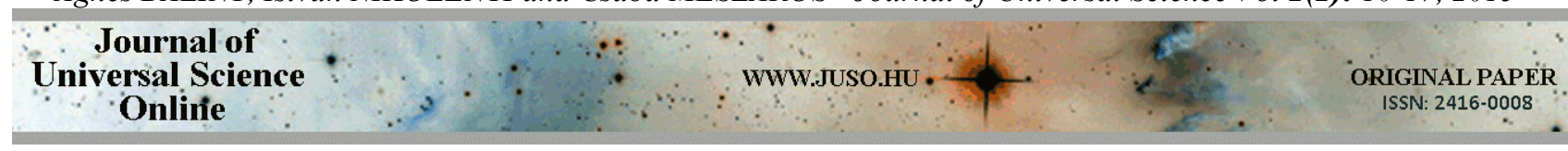

gap in the literature and to lay down mathematical foundation for the relevant Fourier-analysis formalism to be applied in the elastic-, and inelastic scattering experiments playing crucial role in material structure investigation.

\section{Generalization of the concept of structure factors within frame of the theory of projective representations}

In the present section we give a refinement of the existing formalism of the diffuse X-Ray scattering formalism on the base of the line group formalism. As it is well-known from the classic literature of the topic e.g. [30] for a non-ideal condensed matter system being able to scatter X-rays coherently, the electron density function can be given in the form of

$$
\rho(\vec{r})=\langle\rho(\vec{r})\rangle+\Delta \rho(\vec{r})
$$

where $\langle\rho(\vec{r})\rangle$ denotes the electron density function for the ,averaged part" of the lattice being investigated, while $\Delta \rho(\vec{r})$ is related to fluctuational inhomogeneities. Then, the intensity of the diffracted beams can be explained by use of the Patterson autocorrelation function $P(\vec{r})$ defined e.g. [30] by the convolution operation

$$
P(\vec{r})=\{\langle\rho(\vec{r})\rangle *\langle\rho(-\vec{r})\rangle\}+\{\Delta \rho(\vec{r}) * \Delta \rho(-\vec{r})\},
$$

and its Fourier-transformed form as

$$
I(\vec{\kappa})=\mathfrak{J}\{P(\vec{r})\} \equiv|\bar{F}|^{2}+|\Delta F|^{2},
$$

where $\vec{\kappa}=\vec{k}-\vec{k}_{0}$ is the scattering vector (in an elastic scattering process), while $\bar{F}$ and $\Delta F$ denote the Fourier transformed forms of the functions $\langle\rho(\vec{r})\rangle$ and $\Delta \rho(\vec{r})$, respectively. It must also be pointed out here, that from all of the possible variants of diffusively scattered X-rays, electrons or thermal neutrons from condensed matter systems, we base here our modeling work in agreement with the general formalism also described in a suitable manner by Cowley for extended defects, and particularly for the case of stacking faults. Accordingly, the electrondensity function is given by the following convolution operation:

$$
\rho(\vec{r})=\rho_{0}(\vec{r}) * \mathrm{~d}(\vec{r}),
$$

where $\rho_{0}(\vec{r})$ denotes the electron density function of a layer consisting of scattering centres, while $\mathrm{d}(\vec{r})$ is a distribution function.
The relevant generalized autocorrelation function is given by:

$$
P(\vec{r})=\rho_{0}(\vec{r}) * \rho_{0}(-\vec{r}) * D(\vec{r})
$$




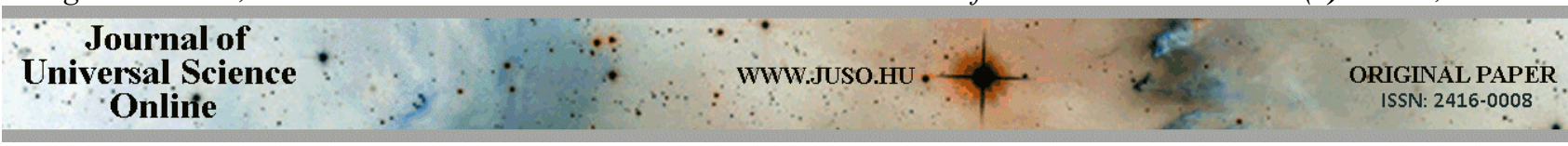

where $D(\vec{r})$ is the generalized autocorrelation function relevant for distribution of layers. It is generally accepted within frame of this formalism, that it can be characterized by
Poisson's type probability distribution functions.

Then, in a more specified manner, for the diffuse scattering intensity contribution we may write after [31]:

$$
|\Delta F|^{2} \equiv I_{D S}(\vec{\kappa})=\sum_{\vec{r}} H(\vec{r}) C(\vec{r}) \cdot \mathrm{e}^{-2 \pi i \vec{k} \vec{r}},
$$

where $H(\vec{r})$ denotes the supersymmetry function, for which we also postulate here, that it is invariant against symmetry transformations from a group having generalized translations, too (i.e. from a given line group) and which can be therefore represented in a Fourier series form as: $H(\vec{r})=\sum_{\vec{h}} T_{\vec{h}} \cdot e^{2 \pi i \vec{h} \vec{r}}$.

The function $C(\vec{r}) \sim e^{-\lambda r}$ is the so-called spatial correlator realizing randomization of the superstructure being investigated, and whose Fourier-transformed form is of Lorentzian shape (if we accept - following again Naish and Grebennikov - the correlator to be of exponentially decaying character, with characteristic correlation length $\left.\xi \equiv \lambda^{-1}\right)$ :

$$
\mathfrak{I}\{C(\vec{r})\} \equiv \tilde{C}(\vec{\kappa})=\frac{\frac{1}{2} \operatorname{sh}(\lambda)}{\sin ^{2}(\lambda / 2)+\sin ^{2}(\pi \kappa)} \equiv \Lambda(\vec{\kappa}, \lambda) .
$$

Then, the diffuse scattering intensity contribution is given by:

$$
I_{D S}(\vec{\kappa})=\sum_{\vec{h}} T_{\vec{h}} \cdot \tilde{C}(\vec{\kappa}-\vec{h}) \equiv \sum_{\vec{h}} T_{\vec{h}} \cdot \Lambda(\vec{\kappa}-\vec{h}, \lambda)=\sum_{\vec{h}} T_{\vec{h}} \cdot \frac{\frac{1}{2} \operatorname{sh}(\lambda)}{\sin ^{2}(\lambda / 2)+\sin ^{2}[\pi(\kappa-h)]} .
$$

In an earlier paper of ours [15], we supposed validity of the following simple type function with respect to symmetry transformations from a given line group $\underline{L}$ : invariance properties of the supersymmetry

$$
\left(R \mid \vec{v}_{R}+\vec{t}\right) \equiv g \in L \Rightarrow \hat{D}(g) H(\vec{r})=H(\vec{r}) .
$$

According to the basic new concept being elaborated in the present paper, the above given simple invariance relation (12) must be generalized in the sense of projective representations. Accordingly (see also the Appendix in this respect), we will have:

$$
\left(R \mid \vec{v}_{R}+\vec{t}\right) \equiv g \in L \Rightarrow \hat{D}(g) H(\vec{r})=\varepsilon_{g} H(\vec{r}),
$$

i.e., the relevant projective representations of a given line group must be elaborated in detail and applied. 


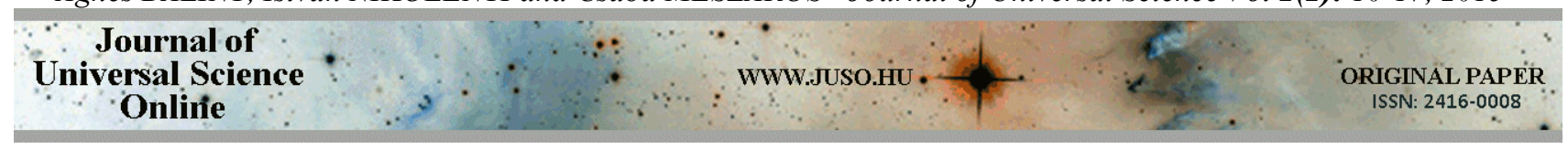

\section{Appendix}

In this Appendix, we summarize briefly some basic features of projective representations of groups after [26]. Accordingly, in the case of projective representation of a group $G$, the basic homomorphism relation relevant for a representation $D(G)$ of its, i.e.

$$
\begin{gathered}
\mathrm{D}(g) \mathrm{D}(h)=\mathrm{D}(g h),(\forall g, h \in G), \\
\text { must be replaced by } \\
\mathrm{D}(g) \mathrm{D}(h)=\varepsilon_{g, h} \mathrm{D}(g h),\left(\forall g, h \in G:\left|\varepsilon_{g, h}\right|=1\right) .
\end{gathered}
$$

Then, from relation of the associative law for group elements $(f \cdot g) \cdot h=f \cdot(g \cdot h),(\forall f, g, h \in G)$, we then get directly for the phase factors:

$$
\varepsilon_{f, g} \cdot \varepsilon_{f g, h}=\varepsilon_{f, g h} \cdot \varepsilon_{g, h} \cdot
$$

It can also be directly seen, that general representations of the Möbius group obey exactly the same system of relations, i.e. they are de facto projective representations.

Namely, if we would like to connect the concept of projective representations to the Bessel's function formalism necessary for description of the X-Ray scattering from ideal
Q1D systems within frame of kinematic approximation e.g. [4], [30], it is necessary to study bilinear transformations leaving invariant the unit complex circle (whose center is identical with the origin of a complex plane $z$ ). Therefore, the following type of transformations must be applied:

$$
\mathrm{z} \mapsto \mathrm{w}(z)=\frac{a z+b}{c z+d},(a d \neq b c)
$$

$(a, b, c, d$ are in general case all complex numbers), mapping the unit circle $|z|=1$ conformally into $|w|=1$, i.e. into another one unit circle. These types of transformations may be combined and form a group of Möbius' (or: homographic type) transformations

\section{Conclusions}

The projective representations of line groups are proposed for detailed analyses of crystals with incommensurately modulated structures. It is shown, that use of projective representations does not lead to contradictions with the already existing formalisms and may significantly extend their domain of applicability. The relevant new mathematical formulae of structure factors are introduced by use of these representations. Finally, the mathematical formalism of the diffuse scattering of X-rays from modulated 


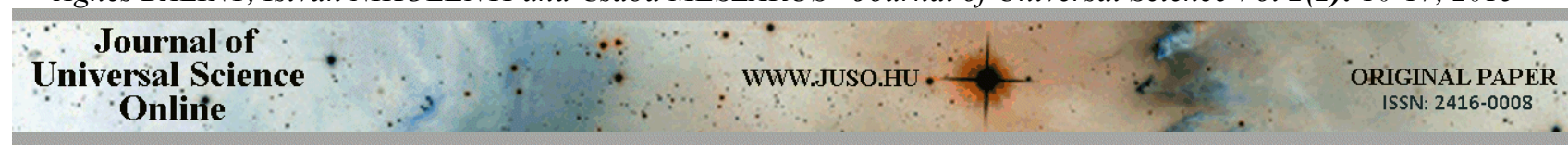

structures is also extended via novel-type formulae of structure factors also generalized by use of projective representations of the exact symmetry groups of quasi-onedimensional systems.

\section{References}

1. Hermann, C.: (1929). XVI. Zur systematischen Strukturtheorie. Zeitschrift für Kristallographie 69(1-6): pp 250-270.

(DOI: 10.1524/zkri.1929.69.1.250)

2. Vujičić, M., Božović, I.B., Herbut, F.: (1977). Construction of the symmetry groups of polymer molecules. Journal of Physics A10 pp 1271-1279.

3. M. Damnjanović; I. Milošević, Line Groups in Physics (Theory and Applications to Nanotubes and Polymers); Springer-Verlag: BerlinHeidelberg, 2010, p 191.

4. B.K. Vainshtein, Diffraction of X-Rays by Chain Molecules; Elsevier Publ. Co.: Amsterdam 1966, p 414.

5. Watson, J.D., Crick, F.H.C.: (1953). A Structure for Deoxyribose Nucleic Acid. Nature 171: pp 737-738.

6. Crick, F.H.C., Watson, J.D.: (1954). The Complementary Structure of Deoxyribonucleic Acid. Proceedings of the Royal Society of London A223: pp 80-96. (DOI: 10.1098/rspa.1954.0101.)

7. Damnjanović, M., Vujičić, M.: (1982). Magnetic line groups. Physical Review B 25: pp 6987-6994. (DOI:

http://dx.doi.org/10.1103/PhysRevB.25.6987)

8. Božović, I.B., Vujičić, M., Herbut, F.:(1978). Irreducible representations of the symmetry gtoups of polymer molecules. Journal of Physics A11: pp 2133-2147.

9. Božović, I.B., Vujičić, M.: (1981). Irreducible representations of the symmetry groups of polymer molecules. II. Journal of Physics A

Mathematical and General 14: pp 777-795.

(DOI: 10.1088/0305-4470/14/4/009)
10. Damnjanović, M., Milošević, I., Vuković, T., Sredanović, R.: (1999). Full symmetry, optical activity, and potentials of single-wall and multiwall nanotubes. Physical Review B 60: pp 2728-2739. (DOI:

http://dx.doi.org/10.1103/PhysRevB.60.2728)

11. Barros, E. B., Jorio, A., Samsonidze, G.G., Capaz, R.B., Souza Filho, A.G., Mendes Filho, J., Dresselhaus. G., Dresselhaus, M.S.: (2006). Review on the symmetry-related properties of carbon nanotubes. Physics Reports 431(6): pp 261-302. (DOI:10.1016/j.physrep.2006.05.007)

12. Vuković, T., Damnjanović, M.: (2007). Diffraction intensity and symmetry of single-wall carbon nanotubes. Nanotechnology 18(37): pp 375708.

13. Mészáros, Cs., Bánkuti, J.: (1994). Line groups and modulated systems. Translational invariance in modulated systems. Physica Status Solidi B 183 (1): pp 73-77. (DOI: $10.1002 /$ pssb.2221830104)

14. Vuković, T., Milošević, I., M. Damnjanović, M.: (1996). Molien functions and commensurability of the helicoidal ordering. Physics Letters A 216(6): pp 307-312. (DOI:10.1016/0375-9601(96)002939)

15. Mészáros, Cs., Bálint, Á., Kirschner, I., Laiho, R., Lähderanta, E., Bánkuti, J.: (1997). Modelling of the oxygen ordering in $\mathrm{YBa} 2 \mathrm{Cu} 3 \mathrm{O} 7-\mathrm{x}$ by line group technique. Superlattice and Microstructure 21(3): pp 381-388. (DOI:10.1006/spmi.1996.0405)

16. Kirschner, I., Mészáros, Cs., Laiho R.: (1997). Symmetry analysis of modulated solid systems by line group method. Zetschrift für Physik B 104(2): pp 289-293. (DOI: $10.1007 / \mathrm{s} 002570050451)$ 


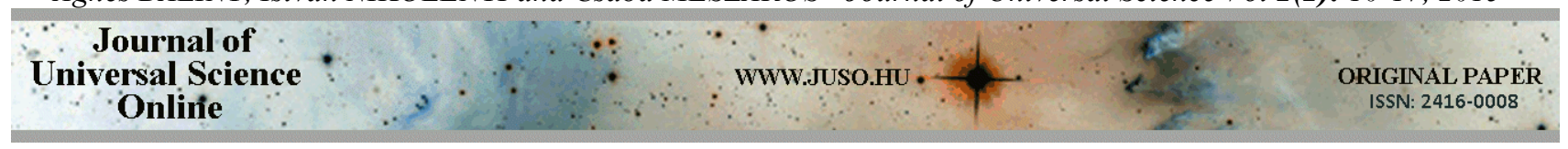

17. Kirschner, I., Mészáros, Cs., Laiho, R.: (1998). Line group theory of commensurate and incommensurate modulations. European Journal of Physics B 2(2): pp 191-196. (DOI: http://dx.doi.org/10.1007/s100510050240)

18. Kirschner, I., Mészáros, Cs.: (2001). Symmetry analysis of static soliton structures and elementary excitations in incommensurately modulated crystals. Journal of Physics: Condensed Matter 13 (23): pp 5399-5411.

19. Peyrard, R.: (2004). Nonlinear dynamics and statistical physics of DNA. Nonlinearity 17: pp R1-R40.

20. Kocsis, A., Swigon, D.: (2012). DNA stretching modeled at the base pair level: Overtwisting and shear instability in elastic linkages. International Journal of Non-Linear Mechanics 47: pp 639654. (DOI:10.1016/j.ijnonlinmec.2011.10.008)

21. R.Z. Sagdeev, D.A. Usikov, G.M. Zaslavsky, Nonlinear Physics (From the Pendulum to Turbulence and Chaos) Contemporary Concepts in Physics, Harwood Academic Publishers: Chur, London, Paris, New York, Melbourne, 1988; Vol.4., p 675.

22. Todorović, D., Ristovski, L.J., B.S. Tošić, B.S.: (1995). On the Problem of the Existence of Davydov Solitons. Physica Status Solidi B 190: pp 251-259. (DOI: 10.1002/pssb.2221900133)

23. H.W. Streitwolf, Gruppentheorie in der Festkörperphysik, Akademische Verlagsgesellschaft: Geest \& Portig, Leipzig, 1967, p 222.
24. S.L. Altmann, Induced Representations in Crystals and Molecules, Academic Press: London - New York - San Francisco, 1977, p 369.

25. G. L. Bir, G. E. Pikus, Symmetry and StrainInduced Effects in Semiconductors, John Wiley and Sons: New York, N.Y. ; Toronto : Jerusalem ; London, 1974, p 484.

26. M. Hamermesh, Group Theory and Its Applications to Physical Problems, Dover Publ. Inc.: New York, 1989, p 509.

27. L.D. Landau, E.M. Lifshitz, Statistical Physics 1. $3^{\text {rd }}$ Ed., Butterworth-Heinemann: Oxford, 1980, $\mathrm{p}$ 544.

28. Bálint, Á., Mészáros, Cs.: (2014). Some basic mathematical features of the simultaneous convection anomalous diffusion transport processes. Journal of Universal Science Online 1:(1): pp 1-5.

29. Wu, G.Ch.: (2010). A Fractional Lie Group Method For Anomalous Diffusion Equations Communications on Fractional Calculation 1, $27-31$.

30. J.M. Cowley, Diffraction Physics, North Holland: Amsterdam, 1975, p 430.

31. Grebennikov, V.I., Naish, V.E.: (1991). Intensity of diffusively scattered X-rays from chaotized superstructures. Physics of Metals and Metallography 71: pp 124-132.

32. Marvel, S.A., Mirollo, R.E., Strogatz, S.H.: (2009). Identical phase oscillators with global sinusoidal coupling evolve by Möbius group action. Chaos 19(4): pp 043104-1-11. (DOI: $10.1063 / 1.3247089)$ 